\title{
Neuropathology provides new insight in the pathogenesis of the sudden infant death syndrome
}

\author{
Hannah C. Kinney
}

Received: 15 January 2009 / Accepted: 22 January 2009 / Published online: 10 February 2009

(C) Springer-Verlag 2009

\section{Introduction}

The sudden infant death syndrome (SIDS) is defined as the sudden, sleep-related death of an infant less than 12 months of age that remains unexplained after a complete autopsy, death scene investigation, and review of the clinical history [35]. Typically, a seemingly healthy infant is unexpectedly found dead after a sleep period. Despite a dramatic reduction in the overall rate with national campaigns advocating the supine (back) sleep position [43], SIDS remains the leading cause of postneonatal infant mortality and the third leading cause of infant mortality in general in the United States today [43]. The brain has long been thought to play a critical role in the cause and pathogenesis of SIDS. The recent publication of three excellent articles on brain pathology in SIDS in Acta Neuropathologica indicates that brain research into this major pediatric disorder continues to be important in guiding all SIDS research. Basically, brain research in SIDS follows three directions: (1) analysis to determine a primary (neural) cause of death, (2) analysis to uncover secondary changes that points to the primary cause, either within the brain or in other organ systems, and (3) analysis to determine the age of onset of the chain of events-prenatally and/or postnatally - that results in sudden death in the vulnerable postnatal period. The search for a primary cause is driven by the hypothesis that SIDS is due to intrinsic abnormali-

H. C. Kinney ( $₫)$

Department of Pathology, Enders Building 1112, Children's Hospital Boston, Harvard Medical School, 300 Longwood Avenue, Boston, MA 02115, USA e-mail: Hannah.kinney@ childrens.harvard.edu ties in brain regions critical for the control of arousal and/ or state-dependent respiratory and/or autonomic function that lead to sleep-related death which is precipitated by a homeostatic stressor within a susceptible age-range. Secondary changes in the brain are postulated to result mainly from hypoxia-ischemia complicating repetitive apnea and/or bradycardia prior to the lethal cardiorespiratory event, as well as during the lethal event itself. Finally, the brain disorder is postulated to be developmental in origin due to the parallel onset and occurrence of SIDS with the major critical period in human brain development, i.e., gestation through the first year of life. Yet, despite the compelling rationale for a key role of the brain in SIDS, the brain looks normal under the light microscope, or at the very most, demonstrates seemingly trivial or nonspecific changes. Thus, the challenge for us as neuropathologists in the analysis of SIDS brains - as in the analysis of autistic and schizophrenic brains-is to uncover neurochemical, cellular, and/or molecular abnormalities with the right quantitative tools. The three recent SIDS studies in Acta Neuropathologica cover the spectrum of neuropathological directions in SIDS research with quantitative methodologies: (1) the search for the primary abnormality in the brainstem with the application of an antibody to the serotonin $1 \mathrm{~A}\left(5-\mathrm{HT}_{1 \mathrm{~A}}\right)$ receptor by Machaalani et al. [37], (2) the determination of secondary hypoxic-ischemic changes in the cerebral cortex and hippocampus with MAP2 immunostaining by Oehmichen et al. [49], and (3) the report of critical evidence for a developmental origin of brain pathology, i.e., increased leptomeningeal neurons in the brainstem by Rickert et al. [62]. In the following commentary, I highlight the new insights that these studies bring to our understanding of the brain's role in SIDS in the context of previous neuropathologic and other SIDS studies. 


\section{The search for primary brain causes of SIDS}

While there are many regions throughout the brain that influence arousal or respiratory and autonomic function (e.g., hypothalamus, amygdala), the focus of SIDS research in search of the primary cause has historically focused upon the brainstem. Two decades ago, Hunt and Brouillette [20] articulated the consensus of the scientific community at the time in a seminal review article: "Although numerous general theories of cause are probably worthy of further clinical study, the most compelling hypothesis continues to be that SIDS is related to a brainstem abnormality in the neuroregulation of cardiorespiratory control". The rationale for the SIDS brainstem hypothesis is based upon three main lines of evidence: (1) established human and animal data that the brainstem plays a critical role in respiratory and autonomic regulation, sleep, and arousal [63], i.e., in the primary physiological processes considered abnormal in SIDS infants, (2) analogy to human entities of sleep-related sudden death in children or adults in which isolated or primary pathology is found by neuroimaging and/or at autopsy in the brainstem [55], and (3) reports of subclinical defects in cardiorespiratory control and/or arousal that are consistent with brainstem dysfunction in infants who are studied prospectively and subsequently die of SIDS [24, 25, 31, 60, $66,67,71,77]$. These defects include impaired autoresuscitation (gasping), abnormal respiratory patterning, episodic obstructive apnea during sleep, autonomic dysfunction (episodic tachycardia/bradycardia, abnormal heart rate variability), and arousal deficits, as well as potential impaired mechanisms that maintain homeostasis during fluctuations in heart rate and blood pressure as the infant transitions among REM, NREM, and waking states during the night or nap-time $[24,25,31,60,66,67,71,77]$. In the decade following the review by Hunt and Brouillette, major risk factors for SIDS were discovered, including prone sleep position, face-down position, covered face in the supine position, soft bedding, bed sharing, over-bundling, elevated room temperature, and minor infection around the time of death $[43,71,77]$. The association of SIDS with these risk factors underscores the validity of the brainstem hypothesis because they are exogenous stressors that trigger asphyxia, hypoxia, hypercapnia, thermal imbalance, and/or cardiovascular instability which in turn require intact brainstem defense systems to protect against lethal consequences. These defense systems involve central chemosensitivity to carbon dioxide and oxygen, autoresuscitation, and arousal with head lifting and turning to escape asphyxiating microenvironments via gaining fresh air and/or crying [25, 31, $60,67,71,77]$.

Brainstem research in SIDS has focused upon the specific hypothesis that it is due to a neurotransmitter defect in brainstem respiratory and/or autonomic pathways. Neuro- chemical studies utilizing mainly tissue receptor autoradiography and immunocytochemistry implicate abnormalities in various neurotransmitters in different brainstem regions of SIDS cases. The neurotransmitters include acetylcholine via muscarinic and nicotinic receptors [10, 29, 39, 44], catecholamines [34, 40, 48], Substance P [23, 79], opioids [28], glutamate (via NMDA and non-NMDA receptors) [38, 52], and serotonin (5-HT) [32, 51, 53, 57, 64]. Several of these neurotransmitter studies indicate neurochemical abnormalities in regions which are involved in respiratory and autonomic control in SIDS cases, although the same region has not typically been studied with more than one transmitter marker in the same case, and not all of the abnormalities have been confirmed by independent investigators. In 2000, our group reported abnormalities in 5-HT receptor binding in SIDS cases $(n=52)$ compared to acutely ill controls $(n=15)$ with the broad radioligand ${ }^{3} \mathrm{H}$ lysergic acid diethylamide (which binds to multiple 5-HT receptor subtypes, including $5-\mathrm{HT}_{1 \mathrm{~A}}$ ) [57]. The affected nuclei were located mainly in the medulla oblongata and comprised 5-HT cell bodies, i.e., the raphé (raphé obscurus), extra-raphé (paragigantocellularis lateralis, gigantocellularis, intermediate reticular zone), and ventral medullary rim (arcuate nucleus) - the so-called medullary 5-HT system or network [53]. This caudal 5-HT system is postulated to modulate and integrate diverse homeostatic functions according to the level of arousal via widespread brainstem and spinal cord projections, as opposed to the rostral 5-HT system in the upper pons and midbrain that help modulate cognition, mood, and waking via diffuse forebrain projections $[5,8,11,17,18,36,47,58,61,68,72]$. In a second dataset consisting of SIDS $(n=23)$ and autopsy control $(n=6)$ infants in a high-risk population for SIDS, i.e., the American Indians in the Northern Plains, we confirmed the same 5-HT receptor binding abnormalities as found in the initial dataset [32]. In a third independent dataset comprised SIDS $(n=31)$ and control infants $(n=10)$, we demonstrated multiple 5-HT abnormalities in the 5-HT medullary system of the same SIDS cases, including: (1) reduced $5-\mathrm{HT}_{1 \mathrm{~A}}$ receptor binding in nuclei that contain the 5 -HT cell bodies and those that receive 5-HT projections, e.g., nucleus of the solitary tract, (2) increased 5-HT cell number and density, (3) morphological immaturity of the 5-HT neurons, and (4) reduced 5-HT transporter binding relative to 5-HT cell number [57]. Eighty-seven percent of the SIDS cases in this study were at risk from having medullary 5-HT abnormalities and were exposed to an exogenous stressor at the time of death. Notably, $77 \%$ of the SIDS cases slept in non-recommended sleep positions or environments at the time of death, i.e., they slept prone or on their side, shared a bed, or both. There was also a significantly lower density of $5-\mathrm{HT}_{1 \mathrm{~A}}$ receptor binding in male compared with female SIDS cases $(P=0.038)$, 
thereby providing neurochemical data that may help explain the increased risk (2:1) of SIDS in males compared to females [57]. The main findings in the medullary 5-HT system in SIDS cases led us to the hypothesis that an important subset of SIDS (approximately 70\%) is due to abnormalities in this homeostatic system that lead to compromised protective reflexes and impaired responses to asphyxial, hypercapnic, hypoxic, thermal, and/or cardiovascular challenges in risky sleep environments.

While our group has replicated 5-HT defects in the medulla in three non-overlapping datasets over the last 20 years, confirmation is needed by an independent laboratory utilizing the same or different techniques in order to account for potential inherent biases in a single laboratory, as well as for unrecognized demographic, technical, or other errors. In 2002, Ozawa et al. [51] reported reduced 5$\mathrm{HT}_{1 \mathrm{~A}}$ and $5-\mathrm{HT}_{2 \mathrm{~A}}$ expression with immunocytochemistry in a Japanese dataset of SIDS and controls. The article by Machaalani et al. [37] in the Acta Neuropathologica is significant because it confirms 5-HT-related abnormalities in the medulla in SIDS infants in a rigorous quantitative study with a large sample size, and it relates these abnormalities to important risk factors for SIDS. The investigators tested the specific hypothesis that $5-\mathrm{HT}_{1 \mathrm{~A}}$ immunoreactivity is reduced in the medulla in SIDS cases compared to controls [37]. The investigators applied immunocytochemistry with an antibody to the 5- $\mathrm{HT}_{1 \mathrm{~A}}$ receptor and quantitated the percent of immunopositive neurons (number of immunopositive neurons divided by total number of positive- and negative-immunopositive neurons) in eight nuclei at a standardized rostral and caudal level of the medulla in 67 SIDS infants and 25 controls adjusted for postconceptional age [37]. They observed a reduced percent of immunopositive neurons in the caudal inferior olive and rostral dorsal motor nucleus of the vagus (DMX), nucleus of the solitary tract, cuneatus nucleus, vestibular nucleus, and inferior olive; no differences were found in the raphé (raphé obscurus and raphé pallidus combined) or arcuate nucleus at the ventral surface [37]. In this study, quantitation is based upon the percent of neurons with 5- $\mathrm{HT}_{1 \mathrm{~A}}$ immunostaining; therefore, the finding of a reduced percent of immunopositive neurons indicates a complete reduction in $5-\mathrm{HT}_{1 \mathrm{~A}}$ receptor expression per neuron in a subset of neurons, and/or loss of the neuron itself that expresses $5-\mathrm{HT}_{1 \mathrm{~A}}$ receptors. Receptor autoradiography applied by Paterson et al. [57], on the other hand, measures receptor number and binding affinity with the distinction between these two parameters determined by Scatchard analysis typically performed in tissue homogenates rather than sections. The immunocytochemical analysis by Machaalani et al. [37] suggests that receptor protein number is at least a substantial component of the change in receptor binding in SIDS cases detected by autoradiography $[32,53,57]$.
Importantly, both studies of $5-\mathrm{HT}_{1 \mathrm{~A}}$ receptors by Machaalani et al. [37] and Paterson et al. [57] found differences in nuclei that receive projections from the 5-HT source neurons in the medulla, notably the DMX and nucleus of the solitary tract; the vestibular and cuneatus nuclei were measured by Machaalani et al. [37] but not by Paterson et al. [57]. In contrast, Paterson et al. [57] found reduced $5-\mathrm{HT}_{1 \mathrm{~A}}$ binding in the raphe obscurus and arcuate nucleus, and not in the inferior olive, whereas Machaalani et al. [37] found reduced percent of 5-HT immunopositive neurons in the inferior olive but not the raphé and arcuate nucleus. The reason(s) for these discrepancies is unknown, but may reflect differences in the techniques used, as well as currently unrecognized differences in the study populations. The raphé obscurus, the major site of 5-HT cell bodies with widespread projections throughout the medulla and spinal cord, plays a key role in breathing, cardiovascular function, thermal regulation, and chemosensitivity to carbon dioxide [5, 11, 17, 36, 61]. In the study by Paterson et al. [57], additional findings were reported at this site, including increased density and immature morphology of 5-HT neurons and reduced 5-HT transporter binding relative to 5-HT neuronal number, solidifying 5-HT pathology at this pivotal site in the medullary 5-HT system. Of note, Machaalani et al. did not determine the density of 5-HT neurons so that a comparative analysis between the two studies cannot be made in regard to the increased 5-HT neuronal density in the study by Paterson et al. [57].

Both the immunocytochemical study of Machaalani et al. [37] and autoradiographic studies of our group [32, 57] link 5- $\mathrm{HT}_{1 \mathrm{~A}}$ abnormalities with clinical risk factors, particularly 5-HT-related abnormalities of the rostral DMX for the first time with cigarette smoke exposure, bed sharing, and prone sleep position in the study by Machaalani et al. [37], and of the raphé obscurus with male gender in the study by Paterson et al. [57]. It is important to establish a link between pathologic findings and epidemiologic risk factors because this link: (1) provides biologic plausibility to otherwise seemingly incomprehensible public health messages (e.g., "Back-to-Sleep"), thereby strengthening the likelihood that these messages will be followed, (2) provides critical insight into the possible mechanisms of death, and (3) defines potential subsets of SIDS cases, thereby helping to tease out the clinical components of a likely heterogeneous disorder. Parenthetically, polymorphisms in genes related to the 5-HT transporter may increase risk by potentially decreasing 5HT levels at the synapse via increased efficiency of 5-HT reuptake $[46,50,75]$. Nevertheless, the study by Paterson et al. indicates that medullary abnormalities of $5-\mathrm{HT}_{1 \mathrm{~A}}$ receptor binding occur in SIDS cases regardless of whether they sleep prone or supine, bed share, or have the LL (risky) genotype for the 5-HT transporter, suggesting 
that the underlying defect is associated with sudden death irrespective of the simultaneous presence of a known risk factor, and reinforcing the key point that risk factors are not causative by themselves [57].

Despite certain discrepancies, the studies by Machaalani et al. [37] and Paterson et al. [57] —arguably the most extensive studies of 5 - $\mathrm{HT}_{1 \mathrm{~A}}$ receptors in SIDS to datereach the same conclusion that there is a widespread deficiency of $5-\mathrm{HT}_{1 \mathrm{~A}}$ receptors across medullary nuclei in SIDS cases, particularly in nuclei that receive 5-HT projections from raphé and extra-raphé 5-HT source neurons and which are known to influence diverse homeostatic functions in a state-dependent manner. The report of 5- $\mathrm{HT}_{1 \mathrm{~A}}$ receptor abnormalities by Machaalani et al. [37] is of major significance because it substantiates in an independent dataset and with a different technique medullary 5-HT pathology in SIDS. It appears from the brainstem studies in SIDS to date that neurotransmitters other than 5-HT are also involved in the pathogenesis of SIDS [10, 29, 34, 38, 39, $44,48,52,79]$, and that the neurochemical pathology in SIDS brainstems is not isolated to a single neurotransmitter system. Yet, the 5-HT abnormality is the most reproducible and robust neurotransmitter finding to date. It is uncertain how the abnormalities in the medullary 5-HT system translate into a mechanism for sudden and unexpected death in infants, although it must relate to the importance of this and related brainstem systems in multiple homeostatic processes that, if dysfunctional, could in toto cause death. The sequence of pathophysiological events that result in SIDS obviously cannot be totally deciphered from the "snapshots" of anatomic/neurochemical pathology in the postmortem infant brain. Still, such pathology is essential for the generation of immediately relevant hypotheses for testing in experimental systems, particularly as there are no spontaneous animal models of SIDS or available biomarkers to identify and study living infants at risk. The importance of direct observations in affected human brains at autopsy cannot be overestimated in driving experimental models for they are grounded in the human entity itself and therefore one step closer to clinical translation.

\section{Secondary changes in the brains of SIDS infants}

A major discovery in SIDS research in the last two decades is that the final lethal event may be preceded by chronic episodes of bradycardia and/or apnea days and even weeks prior to the time of death, as detected by physiological tracings in infants who were monitored prior to death and by various physiological assessments in prospective studies of infants who subsequently died of SIDS [24, 25, 31, 66, 71]. The lethal event itself involves, at least in some cases, a failure in autoresuscitation, i.e., gasping $[60,67]$. In addi- tion, the clinical history of at least one acute life threatening event (ALTE) is significantly higher in SIDS cases (12\%) compared to controls (3\%) [59]. The possibility that defects in autonomic and respiratory regulation are linked specifically to medullary 5-HT abnormalities is substantiated by the case report by us of a 2-week-old boy who died of SIDS and was found to have medullary 5-HT abnormalities at autopsy; this same infant was documented prospectively to have subclinical autonomic and respiratory dysfunction at 2 days of age [31]. These combined clinical observations raise important questions for the neuropathologist: is there evidence of hypoxic-ischemic injury in SIDS brains that supports the likelihood that SIDS involves chronic episodic hypoxia-ischemia? Does the agonal event itself, e.g., defective gasping, cause hypoxic-ischemic brain damage?

In 1976, Dr. Richard Naeye published a landmark report of subtle gliosis in the medullary reticular formation in SIDS infants as it pointed to chronic and/or remote injury in infants who were historically considered "normal" until the acute, lethal event, given that gliosis takes days to develop following an insult. Naeye [45] attributed gliosis in SIDS infants to chronic hypoxia complicating sleep apnea and alveolar hypoventilation. Subsequent neuropathologic studies confirmed subtle gliosis in different medullary and pontine but not mesencephalic nuclei [27, 69], including the inferior olive [27]. An important line of evidence supporting hypoxia-ischemia as the cause of the gliosis is the demonstration that its degree of severity and spatial distribution in SIDS cases are similar to that in infants dying with congenital heart disease with clinically demonstrated hypoxia-ischemia [69]. In addition, postmortem arteriograms of the human medulla demonstrate a close relation between the nuclei with gliosis in SIDS brainstems and the end zones of perforating arteries, suggesting a role for hypoperfusion, perhaps complicating bradycardia and apnea [69]. The reports in SIDS cases of subtle, albeit non-specific, gliosis in the brainstem is of particular interest, as this structure is a recognized region of vulnerability to hypoxiaischemia in early life, particularly the inferior olive [54]. In addition, apoptosis has been reported in brainstem nuclei of SIDS cases, consistent with hypoxia-induced programmed cell death [74] Importantly, histopathologic abnormalities consistent with hypoxia-ischemia have also been reported in regions rostral to the brainstem, including reactive microglia in the hippocampus [9], increased number of copper/zinc superoxide dismutase- and glutathione peroxidaseexpressing neurons in the hippocampus [19], cerebral white matter gliosis [26], and periventricular leukomalacia [26, 70]. While forebrain and brainstem abnormalities have yet to be reported in the same SIDS cases in a single study, the topography and cellular features in combination are consistent with hypoxic-ischemic injury in susceptible regions in the human infant brain, i.e., brainstem, hippocampus, and 
cerebral white matter. The possibility of chronic intermittent hypoxia prior to death is further supported by reports in SIDS cases of other "hypoxic biomarkers", i.e., hyperplasia of pulmonary neuroendocrine cells [7], reduced hypoxanthine oxidase in the vitreous humor [73], and elevated vascular endothelial growth factor in the cerebrospinal fluid [22].

The article by Oehmichen et al. [49] in Acta Neuropathologica is an extension of the distinguished line of neuropathologic studies in search of irrefutable evidence for the hypothesis that SIDS is associated with hypoxic-ischemic brain injury. The unstated premise of this study, similar to previous brain studies of hypoxic-ischemic markers in SIDS [53, 69], is that pathological change in SIDS cases is due to hypoxia-ischemia if its topography and cellular/subcellular features mimic those in the brains of autopsied infant cases with clinically known hypoxia-ischemia, e.g., infants dying of congenital heart disease or accidental asphyxia. Oehmichen et al. [49] applied for the first time the marker for hypoxia-ischemia of microtubule associated protein 2 (MAP2). Antibodies to this cytoskeleton phosphoprotein demonstrate the somatodendritic compartment of virtually all neurons but pyramidal neurons in particular [4, 49], as MAP2 regulates the dynamic cytoplasmic assembly of microtubules and provides the scaffolding for organelle distribution and localization of signal transduction in dendrites, particularly near spines [4]. The investigators here use an antibody against MAP2 as a sensitive probe of hypoxic-ischemic injury as reductions in the intensity of MAP2 immunostaining are associated with various hypoxic-ischemic conditions in human autopsied brain, including with hyper-acute insults that precede morphological changes in neurons [4, 49]. The percentage of MAP2immunoreactive neurons (of 100 total neurons counted) were compared among a SIDS group $(n=17)$, a group with known hypoxic-ischemic insult $(n=15)$, and a control group with a known cause of death (non-SIDS) and no known hypoxia-ischemia $(n=10)$. Oechmichen et al. found a generalized trend in reduced percent of MAP2immunostained neurons in the SIDS and hypoxic-ischemic cases versus the control group, suggesting that the brain findings in the SIDS cases mimicked those of the hypoxicischemic group and therefore had a similar if not identical insult, i.e., hypoxia-ischemia. Yet, the mimicry is not complete, as the reduction in percent MAP2-immunoreactive neurons was more severe in the frontal cortex in the hypoxic-ischemic group compared to the SIDS group, reaching statistical significance in the hypoxic-ischemic group but not in the SIDS group compared to the control group. In addition, the percent of MAP2 immunopositive neurons were significantly reduced in CA4, as well as CA2 and $\mathrm{CA} 3$, in the hippocampus in the hypoxic-ischemic group compared to controls, but was only significantly reduced in
$\mathrm{CA} 2$ and CA3, and not CA4, in the SIDS cases. The differences between the SIDS and hypoxic-ischemic groups may reflect in part with the variability in disease entities in the hypoxic-ischemic group with unavoidable differences in the timing, length, and intensity of hypoxia-ischemia. A key lesson of this study is that all infant deaths, e.g., those due to the entities in the control group, are not associated with (agonal or other) hypoxic-ischemic change, and therefore the demonstration of positive "hypoxic" tissue markers in SIDS cases tells us that the mechanism of death involves hypoxia-ischemia which is not necessarily universal to the death process itself.

The question remains - could hypoventilation be the primary inciting event in SIDS and could there be an intrinsic brainstem defect in protective responses to hypoxia and/or hypercarbia that leads to lethal asphyxia in a sleep situation that would be non-fatal in a normal infant? The risk factors for SIDS suggest scenarios of dangerous sleep environments that could lead to the homeostatic stressors of asphyxia, and hypoxia, e.g., rebreathing exhaled gases or upper airway compression in the face-down (prone) position. The findings of abnormalities in the medullary 5-HT system by Machaalani et al. [37] and others [32, 51, 53, 57] raise the possibility that these and other neurotransmitter abnormalities are the primary defect in SIDS that account for a failure of protective mechanisms to homeostatic derangements impinging upon the infant during a sleep period. In this regard, we found that 5-HT receptor binding patterns are not the same in SIDS cases as those in cases who die with severe oxygenation disorders, arguing against the possibility that hypoxia-ischemia per se is the cause of the 5-HT receptor binding abnormalities [32]. In a piglet model of intermittent hypercapnic hypoxia, however decreased $5-\mathrm{HT}_{1 \mathrm{~A}}$ receptor immunoreactivity occurs in the DMX, as found in SIDS infants [37, 65]; while this neurochemical change may be a secondary "pathologic response" to hypercapnic hypoxia, we raise the possibility that it is part of the "adaptive response" by this nucleus to the insult, and that this adaption may be inadequate in SIDS infants, perhaps due to a combination of maladaptive changes in the multiple nuclei that contain 5-HT neurons and/or receive 5HT terminals and demonstrate 5-HT abnormalities. This idea is based upon the recognition that 5-HT is critically involved in synaptic adjustments in response to hypoxia [2]. Long-term facilitation (LTF) is an enhancement of ventilation or respiratory motor output (measured in respiratory nerve or hypoglossal discharge) that persists for hours after intermittent hypoxia and is considered an adaption to hypoxia. This adaptive response is mediated by 5 -HT via its release from the caudal raphé to bind to 5-HT receptors (probably $5-\mathrm{HT}_{2 \mathrm{~A}}$ ) in the phrenic nucleus that drives the diagram and in the hypoglossal nucleus that mediates upper airway patency. We suggest that the abnormalities in raphé 
5-HT neurons result, at least in part, in impaired phrenic and/or hypoglossal LTF that in turn compromises the infant's protective response to hypoxia, including in the airway.

\section{The search in the brain for the "onset of SIDS"}

The timing of the origin of SIDS is critical to the determination of its cause(s) and pathogenesis. While SIDS occurs in the first year of postnatal life, with $90 \%$ in the first 6 months, and a peak at 2-4 months, the possibility exists that sudden and unexplained death also occurs in the fetus that is due to the same cause as in the infant. In this regard, unexplained stillbirth, i.e., intrauterine fetal demise occurring $\geq 20$ gestational weeks that remains unknown after examination of the placenta, autopsy, and review of the maternal history, may share, at least in some cases, the same etiology and pathogenesis of SIDS in regards to homeostatic challenges (in the fetus which are not protective entirely by the placenta) [12]. The key evidence that SIDS originates during fetal life is its association with risk factors related to pregnancy [3, 21, 33, 43], as well as with placental pathology [16]. The risk factors include maternal use of alcohol, tobacco cigarettes, heroine, and cocaine during pregnancy $[3,21,43]$, and maternal anemia during pregnancy [33]. Developmental brain research in SIDS is driven by the hypothesis that it is a maturational disorder associated with subtle derangements in neuronal proliferation, migration, and/or differentiation. Reports of developmental abnormalities in SIDS brains include persistence of the external granular layer of the cerebellar cortex, suggestive of defective pre- and postnatal neuronal migration [6], underpopulation of neurons in the inferior olive in the medulla [30], and hypoplasia of the arcuate nucleus [14, 42] - all neuronal populations which originate in a common embryonic analage, i.e., the rhombic lip at the dorsal pontomedullary junction. Arcuate nucleus hypoplasia has also been reported in unexplained stillbirth, thereby indicating shared brainstem pathology between this entity and SIDS [41]. The arcuate nucleus is the putative human homolog of the respiratory chemosensitive fields in the ventral medullary surface of experimental animals that mediate responses to carbon dioxide $[13,56]$. Thus, a primary defect in this site may impair protective responses to hypercapnia generated by rebreathing exhaled gases in the face-down position or upper airway occlusion. Other brain findings suggest the possibility that acquired hypoxic-ischemic injury also occurs in SIDS cases during the fetal period, i.e., periventricular leukomalacia [26, 70], a recognized lesion of vulnerable fetal white matter, with a peak at 28-34 gestational weeks, and neuronal necrosis and apoptosis in the arcuate nucleus in unexplained stillbirth, potentially leading to underpopulation of arcuate neurons mimicking hypoplasia [15]. In addition, placental abnormalities with features of maternal vascular underperfusion that can lead to fetal hypoxia have been linked to SIDS [16].

The study by Rickert et al. [62] in Acta Neuropathologica provides compelling evidence for a subtle developmental defect in SIDS brainstems that support a prenatal origin. These investigators quantitated the number and density of leptomeningeal neurons in 15 brain regions in 24 SIDS cases compared to eight controls, and reported a statistically significant increase in these neurons in the brainstem but interestingly not in rostral sites, e.g., cerebral hemispheres. Leptomeningeal neurons result from "breaks" in the glial limitans during neuronal migration for unknown reasons, and thus represent displaced migrating neurons beyond this barrier into the leptomeningeal space. While this unique finding does not specify the nature of the insult, it pinpoints its occurrence to the early fetal period when brainstem neurons are undergoing migration to their final positions-and more importantly, it tells us that an intrauterine insult affects brainstem formation, however, subtly. Could excessive leptomeningeal neurons in the brainstem be a marker of defective developmental mechanisms also at play in systems critical to homeostasis? We do not know the answer to this question yet, but we do have evidence that developmental abnormalities are present in these homeostatic systems, for example, in the arcuate nucleus at the ventral medullary surface $[14,42]$ and in the medullary 5-HT system [57]. In our study of the medullary 5-HT system in SIDS infants among the American Indians of the Northern Plains, we found that 5-HT receptor binding in the arcuate nucleus is significantly lower in infants whose mothers smoke during pregnancy (irrespective of the cause of infant death), as well as marginally lower in infants whose mothers drank during pregnancy, suggesting that prenatal smoking and alcohol exposures adversely affect postnatal 5-HT receptor binding [32]. Furthermore, there is an increased density of 5-HT neurons in the medullary 5-HT system in SIDS cases, and the neurons display a "simple" rather than "complex" morphology, consistent with a developmental impairment in 5-HT neuronal cell number and differentiation [57]. Importantly, the possibility that medullary 5-HT abnormalities are developmental in origin relate to the recognition that the risk factors for SIDS of maternal cigarette smoking and alcohol use during pregnancy impair 5-HT neuronal proliferation, migration, and differentiation in animal models and cell culture systems [78, 80]. Moreover, 5-HT serves as a trophic factor early in embryonic and fetal development prior to synaptic transmission [1] —at these early stages, it directly affects neuronal proliferation, migration, and differentiation. Further evidence for a link between the pathogenesis of SIDS and brain maturation is the association of certain 
polymorphisms in genes responsible for the development of the autonomic nervous systems with SIDS [76].

\section{Conclusions}

The three recent neuropathologic studies in SIDS in Acta Neuropathologica provide exciting new information toward a more complete understanding of the brain's role in SIDS. These studies solidify mounting evidence that SIDS is associated with a brainstem 5-HT defect, cerebral hypoxia-ischemia is involved, and the onset of at least some SIDS cases is during gestation. These studies are important because they are: (1) hypothesis-driven, (2) based upon reproducible quantitative methods, (3) involve relatively large sample sizes, (4) account for postmortem interval, and (5) adjust for gestational, as well as postnatal, age. What is needed now is a unified study of a large number of SIDS and control brains with combined markers to establish brainstem neurotransmitter, hypoxicischemic, and developmental pathology in the same SIDS cases and its relationship to risk factors. A potential scenario that ties together the diverse pathologic features found in different brain studies to date is that SIDS is due to an intrinsic brainstem defect in the defense systems protective against hypoxia, hypercapnia, asphyxia, thermal stress, and/or cardiovascular instability which: (1) originates in utero, (2) is expressed at a subclinical level-or even clinical level, i.e., ALTE_-in the susceptible postnatal period, (3) is triggered by repetitive homeostatic challenges which occur at a sublethal level, (4) results in secondary hypoxic-ischemic injury to the forebrain and brainstem, and (5) proves fatal when progression of the brainstem lesion and/or its cumulative effects reach a critical-point. Indeed, it is unknown if certain infants "survive" the vulnerable period and subsequently develop disorders of 5-HT or related neurotransmitter systems in later life, or alternatively, suffer from the static effects of sublethal hypoxic-ischemic damage to vulnerable forebrain and brainstem regions. Brain studies are needed now more than ever for this scenario and suggest the possibility that SIDS is not acute and perhaps could be prevented if we had the right "markers" to identify living infants at risk and the right drug or other means to intervene. It is hoped that the three recent studies in SIDS brains in Acta Neuropathologica stimulate more neuropathologists to pursue the compelling brain clues, thereby moving SIDS research closer to the determination of its cause and universal prevention.

Acknowledgments The assistance of Mr. Richard A. Belliveau in manuscript preparation is greatly appreciated. The author thanks Dr. George B. Richerson for helpful review of the manuscript.

\section{References}

1. Azmitia EC (2001) Modern views on an ancient chemical: serotonin effects on cell proliferation, maturation, and apoptosis. Brain Res Bull 56:413-424

2. Baker-Herman TL, Fuller DD, Bavis RW et al (2004) BDNF is necessary and sufficient for spinal respiratory plasticity following intermittent hypoxia. Nat Neurosci 7:48-55

3. Blair PS, Fleming PJ, Bensley D et al (1996) Smoking and the sudden infant death syndrome: results from 1993-5 case-control study for confidential inquiry into stillbirths and deaths in infancy. Confidential enquiry into stillbirths and deaths regional coordinators and researchers. BMJ 313:195-198

4. Blomgren K, McRaie A, Elmered A, Bona E, Kawashima S, Saido TCh, Ono T, Hegberg H (1997) The calpain proteolytic system in hypoxia-ischemia. Ann N Y Acad Sci 825:104-119

5. Brown JW, Sirlin EA, Benoit AM, Hoffman JM, Darnall RA (2008) Activation of 5-HT1A receptors in medullary raphe disrupts sleep and decreases shivering during cooling in the conscious piglet. Am J Physiol Regul Integr Comp Physiol 294:RR 884-RR 894

6. Cruz-Sanchez FF, Lucena J, Ascaso C, Tolosa E, Quinto L, Rossi ML (1997) Cerebellar cortex delayed maturation in sudden infant death syndrome. J Neuropathol Exp Neurol 56:340-346

7. Cutz E, Perrin DG, Pan J, Haas EA, Krous HF (2007) Pulmonary neuroendocrine cells and neuroepithelial bodies in sudden infant death syndrome: potential markers of airway chemoreceptor dysfunction. Pediatr Dev Pathol 10:106-116

8. Darnall RA, Harris MB, Gill WH, Hoffman JM, Brown JW, Niblock MM (2005) Inhibition of serotonergic neurons in the nucleus paragigantocellularis lateralis fragments sleep and decreases rapid eye movement sleep in the piglet: implications for sudden infant death syndrome. J Neurosci 25:8322-8332

9. Del Bigio MR, Becker LE (2004) Microglial aggregation in the dentate gyrus: a marker of mild hypoxic-ischaemic brain insult in human infants. Neuropathol Appl Neurobiol 20:144-151

10. Duncan JR, Randall LL, Belliveau RA et al (2008) The effect of maternal smoking and drinking during pregnancy upon (3)H-micotine receptor brainstem binding in infants dying of the sudden infant death syndrome: Initial observations in a high risk population. Brain Pathol 18:21-31

11. Erickson JT, Shafer G, Rossetti MD, Wilson CG, Deneris ES (2007) Arrest of 5HT neuron differentiation delays respiratory maturation and impairs neonatal homeostatic responses to environmental challenges. Respir Physiol Neurobiol 159:85-101

12. Fifer WP, Myers MM (2002) Sudden fetal and infant deaths: shared observations and distinctive features. Semin Perinatol 26:89-96

13. Filiano JJ, Choi JC, Kinney HC (1990) Candidate cell populations for respiratory chemosensitive fields in the human infant medulla. J Comp Neurol 293:448-465

14. Filiano JJ, Kinney HC (1992) Arcuate nucleus hypoplasia in the sudden infant death syndrome. J Neuropathol Exp Neurol 5:394403

15. Folkerth RD, Zanoni S, Andiman SE, Billiards SS (2008) Neuronal cell death in the arcuate nucleus of the medulla oblongata in stillbirth. Int J Dev Neurosci 26:133-140

16. Getahun D, Amre D, Rhodes GG, Demissic K (2004) Maternal and obstetric risk factors for sudden infant death syndrome in the United States. Obstet Gynecol 103:646-652

17. Hodges MR, Tallersall GG, Harris MB, McEvoy SD, Richerson DN, Deneris ES, Johnson RL, Chen ZF, Richerson GB (2008) Defects in breathing and thermoregulation in mice with near complete absence of central serotonin neurons. J Neurosci 28:495-505 
18. Hornung JP (2003) The human raphe nuclei and the serotonergic system. J Chem Neuroanat 26:331-343

19. Huggle S, Hunsaker JC 3rd, Coyne CM, Sparks DL (1996) Oxidative stress in sudden infant death syndrome. J Child Neurol $11: 433-438$

20. Hunt CE, Brouillette RT (1987) Sudden infant death syndrome: 1987 perspective. J Pediatr 110:669-678

21. Iyasu S, Randall LL, Welty TK et al (2002) Risk factors for sudden infant death syndrome among northern plains Indians. JAMA 288:2717-2723

22. Jones KL, Krous HF, Nadeau J, Blackbourne B, Zielke HR, Gozal D (2003) Vascular endothelial growth factor in the cerebrospinal fluid of infants who died of sudden infant death syndrome: evidence for antecedent hypoxia. Pediatrics 111:358-363

23. Jordan D, Kermadi I, Rambaud C et al (1997) Autoradiographic distribution of brainstem substance $\mathrm{P}$ binding sites in humans: ontogenic study and relation to sudden infant death syndrome (SIDS). J Neural Transm 104:1101-1105

24. Kahn A, Groswasser J, Rebuffat E et al (1992) Sleep and cardiorespiratory characteristics of infant victim of sudden death: a prospective case-control study. Sleep 15:287-292

25. Kato I, Franco P, Groswasser J et al (2003) Incomplete arousal processes in infants who were victims of sudden death. Am J Respir Crit Care Med 168:1298-1303

26. Kinney HC, Brody BA, Finkelstein DM, Vawter GF, Mandell F, Gilles FH (1991) Delayed central nervous system myelination in the sudden infant death syndrome. J Neuropathol Exp Neurol 50:29-48

27. Kinney HC, Burger PC, Harrell FE Jr, Hudson RP Jr (1983) 'Reactive gliosis' in the medulla oblongata of victims of the sudden infant death syndrome. Pediatrics 72:181-187

28. Kinney HC, Filiano JJ, Assmann SF et al (1998) Tritiated-naloxone binding to brainstem opioid receptors in the sudden infant death syndrome. J Auton Nerv Syst 69:156-163

29. Kinney HC, Filiano JJ, Sleeper LA, Mandell F, Valdes-Dapena M, White WF (1995) Decreased muscarinic receptor binding in the arcuate nucleus in sudden infant death syndrome. Science 269:1446-1450

30. Kinney HC, McHugh T, Miller K, Belliveau RA, Assmann SF (2002) Subtle developmental abnormalities in the inferior olive: an indicator of prenatal brainstem injury in the sudden infant death syndrome. J Neuropathol Exp Neurol 6:427-441

31. Kinney HC, Myers MM, Belliveau RA et al (2005) Subtle autonomic and respiratory dysfunction in sudden infant death syndrome associated with serotonergic brainstem abnormalities: a case report. J Neuropathol Exp Neurol 64(8):689-694

32. Kinney HC, Randall LL, Sleeper LA et al (2003) Serotonergic brainstem abnormalities in Northern Plains Indians with the sudden infant death syndrome. J Neuropathol Exp Neurol 62:1178-1191

33. Klonoff-Cohen HS, Shrinivasan JP, Edelstein SL (2002) Prenatal and intrapartum events and sudden infant death syndrome. Paediatr Perinatol Epidemiol 16:82-89

34. Kopp N, Chigr F, Denoroy L, Gilly R, Jordan D (1993) Absence of adrenergic neurons in nucleus tractus solitarius in sudden infant death syndrome. Neuropediatrics 24:25-29

35. Krous HF, Beckwith JB, Byard RW et al (2004) Sudden infant death syndrome and unclassified sudden infant deaths: a definitional and diagnostic approach. Pediatrics 114:234-238

36. Lovick TA (1997) The medullary raphe nuclei: a system for integration and gain control in autonomic and somatomotor responsiveness? Exp Physiol 82:31-41

37. Machaalani R, Say M, Waters KA (2009) The serotonergic receptor $1 \mathrm{~A}$ in the sudden infant death syndrome (SIDS) brainstem medulla associated with clinical risk factors. Acta Neuropathol, 4 December [Epub ahead of print]. doi:10.1007/s00401-008-0468-x
38. Machaalani R, Waters KA (2003) NMDA receptor 1 expression in the brainstem of human infants and its relevance to the sudden infant death syndrome (SIDS). J Neuropathol Exp Neurol 62:1076-1085

39. Mallard C, Tolcos M, Leditschke J, Campbell P, Rees S (1999) Reduction in choline acetyltransferase immunoreactivity but not muscarinic-m2 receptor immunoreactivity in the brainstem of SIDS infants. J Neuropathol Exp Neurol 58:255-264

40. Mansouri J, Panigrahy A, Filiano JJ, Sleeper LA, St John WM, Kinney HC (2001) Alpha2 receptor binding in the medulla oblongata in the sudden infant death syndrome. J Neuropathol Exp Neurol 60:141-146

41. Matturi L, Minoli I, Lavezzi AM, Cappellini A, Ramos S, Rossi L (2002) Hypoplasia of medullary arcuate nucleus in unexpected late fetal death (stillbirth deaths); A pathologic study. Pediatrics 109:E43

42. Matturri L, Biondo B, Mercurio P, Rossi L (2000) Severe hypoplasia of medullary arcuate nucleus: quantitative analysis in sudden infant death syndrome. Acta Neuropathol 99:371-375

43. Moon RY, Horne RS, Hauck FR (2007) Sudden infant death syndrome. Lancet 370:1578-1587

44. Nachmanoff DB, Panigrahy A, Filiano JJ et al (1998) Brainstem $3 \mathrm{H}$-nicotine receptor binding in the sudden infant death syndrome. J Neuropathol Exp Neurol 57:1018-1025

45. Naeye RL (1976) Brain-stem and adrenal abnormalities in the sudden-infant-death syndrome. Am J Clin Pathol 66:526-530

46. Narita N, Narita M, Takashima S, Nakayama M, Nagai T, Okado $\mathrm{N}$ (2001) Serotonin transporter gene variation is a risk factor for sudden infant death syndrome in the Japanese population. Pediatrics 107:690-692

47. Nattie EE, Li A, Richerson G, Lappi DA (2004) Medullary serotonergic neurones and adjacent neurones that express neurokinin-1 receptors are both involved in chemoreception in vivo. J Physiol 556:235-253

48. Obonai T, Yasuhara M, Nakamura T, Takashima S (1998) Catecholamine neurons alteration in the brainstem of sudden infant death syndrome victims. Pediatrics 10:285-288

49. Oehmichen M, Woetzel F, Meissner C (2009) Hypoxic-ischemic changes in SIDS brains as demonstrated by a reduction of MAP2 reactive neurons. Acta Neuropathol, 14 November [Epub ahead of print]. doi:10.1007/s00401-008-0459-y

50. Opdal SH, Vege A, Rognum TO (2008) Serotonin transporter gene variation in sudden infant death syndrome. Acta Pediatr 97:861865

51. Ozawa Y, Okado N (2002) Alteration of serotonergic receptors in the brain stems of human patients with respiratory disorders. Neuropediatrics 33:142-149

52. Panigrahy A, Filiano J, Sleeper L et al (1997) Decreased kainate receptor binding in the arcuate nucleus of the sudden infant death syndrome. J Neuropathol Exp Neurol 56:1253-1261

53. Panigrahy A, Filiano J, Sleeper LA et al (2000) Decreased serotonergic receptor binding in rhombic lip-derived regions of the medulla oblongata in the sudden infant death syndrome. J Neuropathol Exp Neurol 59:377-384

54. Panigrahy A, White WF, Rava LA, Kinney HC (1995) Developmental changes in $[3 \mathrm{H}]$ kainate binding in human brainstem sites vulnerable to perinatal hypoxia-ischemia. Neuroscience 67:441-454

55. Parenti A, Macchi V, Snenghi R et al (2005) Selective stroke of the solitary tract nuclei in two cases of central sleep apnoea. Clin Neuropathol 24:239-246

56. Paterson DS, Thompson EG, Kinney HC (2006) Serotonergic and glutamatergic neurons at the ventral medullary surface of the human infant: Observations relevant to central chemosensitivity in early human life. Auton Neurosci 124:112-124

57. Paterson DS, Trachtenberg FL, Thompson EG et al (2006) Multiple serotonergic brainstem abnormalities in sudden infant death syndrome. JAMA 296:2124-2132 
58. Pena F, Ramirez JM (2002) Endogenous activation of serotonin$2 \mathrm{~A}$ receptors is required for respiratory rhythm generation in vitro. J Neurosci 22:11055-11064

59. Platt MW et al (2000) A clinical comparison of SIDS and explained sudden infant deaths: how healthy and how normal? CESDI SUDI Research Group. Confidential Inquiry into Stillbirths and Deaths in Infancy study. Arch Dis Child 82:98-106

60. Poets CF (2004) Apparent life-threatening events and sudden infant death on a monitor. Paediatr Respir Rev 5(Suppl A):S383S386

61. Richerson GB (2004) Serotonergic neurons as carbon dioxide sensors that maintain $\mathrm{pH}$ homeostasis. Nat Rev Neurosci 5:449-461

62. Rickert CH, Groß O, Nolte KW et al (2009) Leptomeningeal neurons are a common finding in infants and are increased in sudden infant death syndrome (SIDS). Acta Neuropathol (in press)

63. Saper CB, Richerson GB, Lumsden A (2008) The brainstem and cranial nerves. In: Kandal ER, Schwartz JH, Jessell TM (eds) Principles of neural science, 5th edn. McGraw-Hill, New York (in press)

64. Sawaguchi T, Patricia F, Kadhim H et al (2003) The correlation between serotonergic neurons in the brainstem and sleep apnea in SIDS victims. Early Hum Dev 75(Suppl):S31-S40

65. Say M, Machaalani R, Waters KA (2007) Changes in serotonergic receptors $1 \mathrm{~A}$ and $2 \mathrm{~A}$ in the piglet brianstem after intermittent hypercapnic hypoxia (IHH) and nicotine. Brain Res 1152:17-26

66. Schechtman VL, Lee MY, Wilson AJ, Harper RM (1996) Dynamics of respiratory patterning in normal infants and infants who subsequently died of the sudden infant death syndrome. Pediatr Res 40:571-577

67. Sridhar R, Thach BT, Kelly DH, Henslee JA (2003) Characterization of successful and failed autoresuscitation in human infants, including those dying of SIDS. Pediatr Pulmonol 36:113-122

68. St-John WM, Leiter JC (2007) Maintenance of gasping and restoration of eupnea after hypoxia is impaired following blockers of \{alpha\}-1 adrenergic receptors and serotonin 5HT2 receptors. J Appl Physiol 104:665-673

69. Takashima S, Armstrong D, Becker L, Bryan C (1978) Cerebral hypoperfusion in the sudden infant death syndrome? Brainstem gliosis and vasculature. Ann Neurol 4:257-262
70. Takashima S, Armstrong D, Becker LE, Huber J (1978) Cerebral white matter lesions in sudden infant death syndrome. Pediatrics 62:155-159

71. Thach B, Tragic, sudden death (2008) Potential and proven mechanisms causing sudden infant death syndrome. EMBO Rep 9:114118

72. Tryba AK, Pena F, Ramirez JM (2006) Gasping activity in vitro: a rhythm dependent on 5-HT2A receptors. J Neurosci 26:26232634

73. Vege A, Chen Y, Opdal SH, Saugstad OD, Rognum TO (1994) Vitreous humor hypoxanthine levels in SIDS and infectious death. Acta Paediatr 83:634-639

74. Waters KA, Meehan B, Huang JQ, Gravel RA, Michaud J, Cote A (1999) Neuronal apoptosis in sudden infant death syndrome. Pediatr Res 45(2):166-172

75. Weese-Mayer DE, Berry-Kravis EM, Maher BS, Silvestri JM, Curran ME, Marazita ML (2003) Sudden infant death syndrome: association with a promoter polymorphism of the serotonin transporter gene. Am J Med Genet 117A:268-274

76. Weese-Mayer DE, Berry-Kravis EM, Zhou L et al (2004) Sudden infant death syndrome: case-control frequency differences at genes pertinent to early autonomic nervous system embryologic development. Pediatr Res 56:391-395

77. Williams SM, Mitchell EA, Stewart AW, Taylor BJ (1996) Temperature and the sudden infant death syndrome. Paediatr Perinat Epidemiol 10:136-149

78. Xu Z, Seidler FJ, Ali SF, Slikker W Jr, Slotkin TA (2001) Fetal and adolescent nicotine administration: effects on CNS serotonergic systems. Brain Res 914:166-178

79. Yamanouchi H, Takashima S, Becker LE (1993) Correlation of astrogliosis and substance $\mathrm{P}$ immunoreactivity in the brainstem of victims of sudden infant death syndrome. Neuropediatrics 24:200203

80. Zhou FC, Sari Y, Powrozek TA (2005) Fetal alcohol exposure reduces serotonin innervation and compromises development of the forebrain along the serotonergic pathway. Alcohol Clin Exp Res 29:141-149 